\title{
Successful Transcatheter Arterial Embolization Using N-Butyl-2-Cyanoacrylate as a Treatment for Refractory Inferior Epigastric Artery Pseudoaneurysm Accompanied by an Enterocutaneous Fistula: A Case Report
}

\author{
Myung Won Song ${ }^{1}$, Chan Park ${ }^{2,}{ }^{,}$, Hyoung Ook Kim ${ }^{2}$ and Byung Chan Lee ${ }^{1}$ \\ ${ }^{1}$ Department of Radiology, Chonnam National University Hwasun Hospital, Hwasun, South Korea \\ ${ }^{2}$ Department of Radiology, Chonnam National University Hospital, Gwangju, South Korea \\ "Corresponding author: Department of Radiology, Chonnam National University Hospital, Gwangju, South Korea. Email: rdparkchan@gmail.com \\ Received 2021 April 02; Revised 2021 May 04; Accepted 2021 June 14
}

\begin{abstract}
Inferior epigastric artery (IEA) pseudoaneurysms are well-known postoperative abdominal complications, which often require proper treatment. Treatment options include surgical ligation, transcatheter embolization, and thrombin injection. Here, we report a rare case of an IEA pseudoaneurysm, accompanied by a postsurgical enterocutaneous fistula. The pseudoaneurysm relapsed after transcatheter coil embolization and percutaneous thrombin injection; it was completely occluded by transcatheter arterial embolization using n-butyl-2-cyanoacrylate. The present case shows that a coexisting enterocutaneous fistula can affect the unresponsiveness of patients with IEA pseudoaneurysm to widely accepted treatments, such as coil embolization and thrombin injection, by creating an inflammatory environment. In such cases, repeated therapeutic trials may be required. Transcatheter arterial embolization using n-butyl-2-cyanoacrylate can be a feasible therapeutic option for patients with refractory IEA pseudoaneurysm, accompanied by an enterocutaneous fistula.
\end{abstract}

Keywords: Therapeutic Embolization, Enterocutaneous Fistula, Interventional Radiology, Pseudoaneurysm, N-Butyl-2-Cyanoacrylate, Ultrasound

\section{Introduction}

An inferior epigastric artery (IEA) pseudoaneurysm is an uncommon postoperative abdominal complication. Surgical ligation, transcatheter embolization, and thrombin injection are possible treatment options for this rare condition (1-3). An enterocutaneous fistula (ECF) is a wellrecognized disorder following abdominal surgeries. An ECF itself is a challenging presentation with a high mortality rate of up to $30 \%(4,5)$. It causes various complications, such as sepsis, malnutrition, and electrolyte imbalance, making its management more difficult. Here, we report a rare case of refractory IEA pseudoaneurysm, accompanied by a postoperative ECF, which was successfully treated with transcatheter arterial embolization (TAE) using n-butyl-2-cyanoacrylate (NBCA).

\section{Case Presentation}

A 59-year-old woman was transferred to our hospital with a suspected postoperative intraabdominal hematoma. She had a complicated medical history of multiple surgeries due to colon perforation and its related complications since a gunshot injury 16 years ago. She had undergone the Hartmann reversal procedure in another clinic eight days earlier. Upon arrival, her laboratory findings included a hemoglobin level of $10.6 \mathrm{~g} / \mathrm{dL}$, a platelet count of $232 \times 10^{3} / \mathrm{uL}$, and a partial thromboplastin time of $27.9 \mathrm{sec}$. The prothrombin time-based international normalized ratio (INR) was also 1.32 , which is slightly above the normal range. The patient was not taking any anticoagulants or antiplatelet drugs. After several days of conservative treatment for stabilization, explorative laparotomy and hematoma removal were performed. 
On postoperative day 17 , the patient presented with hematochezia twice. The abdominal computed tomography angiography (CTA) revealed an acute hematoma in the anterior abdominal wall with a left IEA pseudoaneurysm; the contrast medium extravasated to the adjacent jejunal lumen (Figure 1A - C). Also, a focal wall defect with soft tissue thickening and air bubbles in the anterior abdominal wall along the incision line were observed, with a suspicious fistulous tract connecting to the jejunal loop. Transcatheter angiography was performed through the right common femoral artery under local anesthesia using $2 \%$ lidocaine solution; it indicated a pseudoaneurysm, arising from the small branch of the left IEA.

Additional superior mesenteric artery (SMA) angiogram and abdominal aortogram revealed no collateral vessels feeding the pseudoaneurysm. Microcoil was selected among various embolic materials to avoid organ or skin ischemia, which can occur by unintended occlusion of a non-target artery using embolic agents, such as polyvinyl alcohol particles or NBCA. Next, according to the sandwich technique, superselective embolization was successfully performed using 3 - 2 and 4 - 2 mm Tornado coils (Cook Medical, Bloomington, Indiana, USA) (Figure 1D and E). The subsequent selective left internal thoracic artery angiogram revealed no remaining pseudoaneurysms.

The patient remained hemodynamically stable after TAE. Total parenteral nutrition (TPN), followed by a gradual shift to a fluid diet, was applied to control ECF; the patient had no significant gastrointestinal symptoms associated with ECF. Twenty-four days later, her hemoglobin level decreased to $7.7 \mathrm{~g} / \mathrm{dL}$, and she had stable vital signs. The follow-up abdominal computed tomography (CT) scan revealed a decreased, but remaining hematoma in the anterior abdominal wall (Figure 2A). Doppler ultrasound (US) confirmed a 1-cm pseudoaneurysm within the left abdominal wall hematoma (Figure $2 \mathrm{~B}$ ), and a percutaneous thrombin injection was planned for a minimally invasive treatment. Under US guidance, 900 IU of thrombin was injected into the pseudoaneurysm. The pseudoaneurysm was completely thrombosed without complications (Figure 2C).

A follow-up Doppler US, performed one week after thrombin injection, revealed a 0.6-cm pseudoaneurysm in the left abdominal wall hematoma (Figure 2D). Under local anesthesia, the selective left IEA angiogram showed a remaining pseudoaneurysm at the site of the previous coil embolization (Figure 2E). Next, a superselective embolization was successfully performed with a mixture of NBCA (0.5 cc) and lipiodol (3 cc) (Figure 2F). The comple- tion angiogram indicated the total exclusion of the pseudoaneurysm (Figure $2 \mathrm{G}$ ). An additional selective left internal thoracic artery angiogram revealed no evidence of active bleeding or remaining pseudoaneurysm.

The patient complained of abdominal wall pain for one day since TAE; however, no obvious gastrointestinal or skin problems were observed from that day on. After a few weeks of conservative treatment, the patient was discharged with a significantly decreased abdominal hematoma. The follow-up CT examination showed no recurrence of pseudoaneurysm with visible, but a markedly improved ECF.

\section{Discussion}

Abdominal wall hematoma is most commonly induced by a damage to the IEA, which is susceptible to iatrogenic injuries due to its superficial anatomical position (3). IEA pseudoaneurysm is a rare presentation. Conservative treatment is effective in most cases; however, if the patient is symptomatic or hemodynamically unstable, appropriate management is necessary. Among various possible therapeutic options, transcatheter coil embolization is an effective treatment for pseudoaneurysm, with an overall success rate of $90-100 \%(1,6)$.

US-guided thrombin injection is widely accepted as a successful treatment for iatrogenic pseudoaneurysms, with a reported success rate of 75 to $>90 \%(1,3,7)$. In our case, although total occlusion was immediately achieved by both coil embolization and thrombin injection, pseudoaneurysm recurred. There are several possible causes of unresponsiveness to treatment. According to a study by Shieman et al. (7), an underlying occult vessel laceration or vessel infection can cause pseudoaneurysm recurrence after a percutaneous thrombin injection. Thrombomodulin, expressed by a damaged endothelium at the vessel laceration site, activates the anticoagulation pathway and leads to spontaneous thrombolysis, resulting in unresponsiveness to thrombin injection therapy (8). This potentially explains our findings, since our patient had a recent history of abdominal surgery, which might have caused IEA laceration. Hematoma might have also acted as a nidus of infection, triggering vessel infection.

Importantly, our patient presented with ECF at the same time as the IEA pseudoaneurysm was first detected in the CT examination. ECF is a feared postsurgical complication with high morbidity and mortality rates $(5,9)$. The 

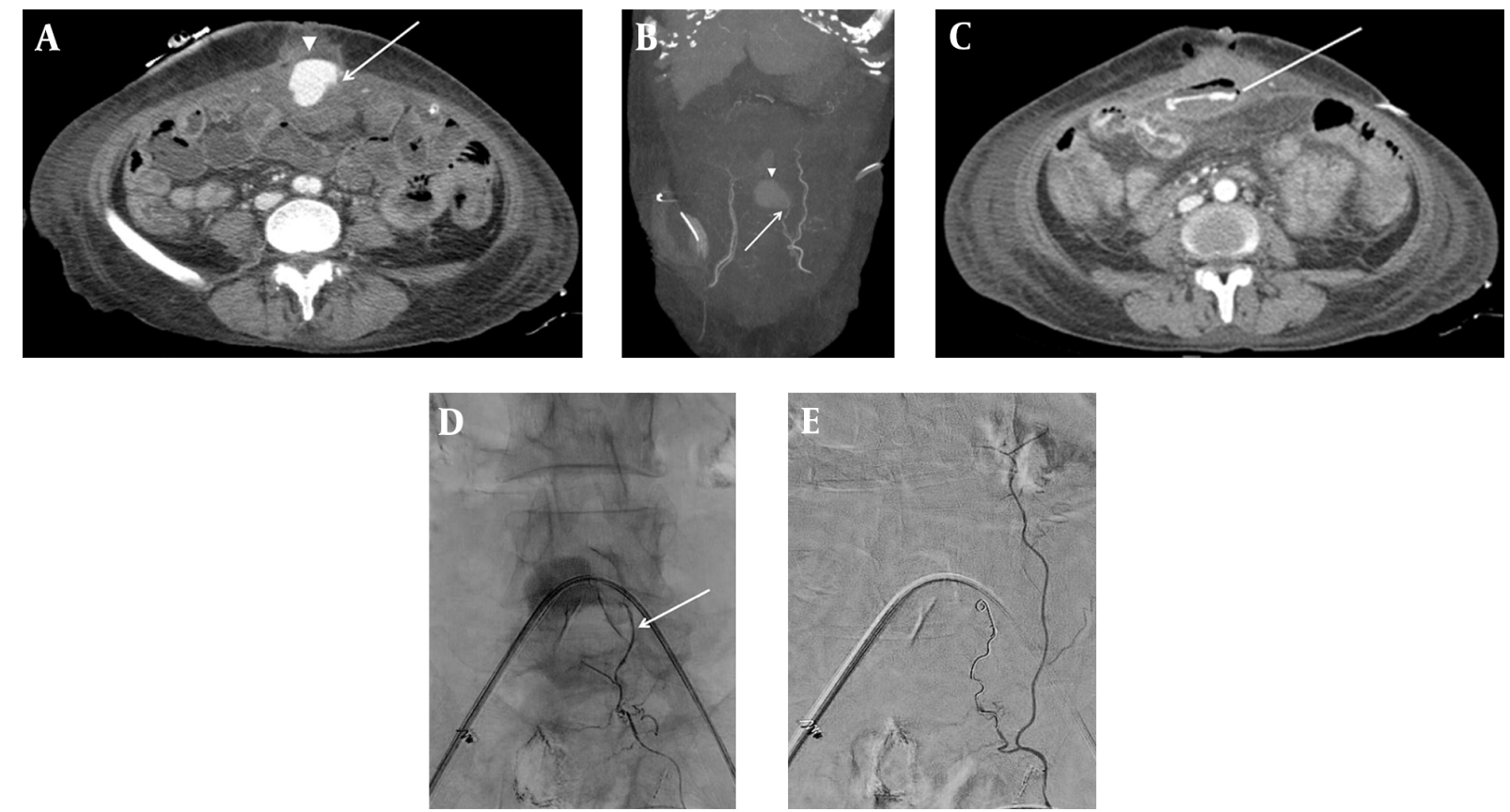

Figure 1. Contrast-enhanced abdominal CT (A) axial image and (B) maximum intensity projection (MIP) image demonstrate acute hematoma (arrowhead) in the paramedian anterior abdominal wall, arising from the left inferior epigastric artery (IEA)(arrow). (C)The contrast medium is extravasated (line) into the adjacent jejunal lumen. The superselective left IEA angiogram (D) shows contrast pooling at the branch of the left IEA (arrow). The completion angiogram after coil embolization (E) shows the disappearance of contrast pooling.

majority of iatrogenic ECFs are due to anastomotic leakage or dehiscence, and spontaneous ECF is thought to be mainly induced by inflammatory processes (4). Also, ECF itself can induce perilesional inflammation by succus and bacteria (5). Although low-output ECF was observed in our case, it might have induced vascular infection, leading to pseudoaneurysm recurrence and increased rebleeding.

TAE using NBCA is an effective treatment for pseudoaneurysms $(2,10)$. NBCA, as a fast-acting permanent embolic agent, enables simultaneous hemostasis of the bleeding focus and collateral vessels. Some characteristics of NBCA can explain the successful outcomes of our case. The operator could control the polymerization time by mixing NBCA with ethiodized oil at different ratios to avoid proximal occlusion in case of superselection difficulty due to anatomical tortuosity (10). This approach also enables effective embolization in patients with a coil embolization history in whom microcoils cannot reach the neck or inflow/outflow vessels in the pseudoaneurysm (2). Our patient had already undergone coil embolization; therefore, NBCA was an appropriate option for the recurred pseudoaneurysm. Also, NBCA is preferred for coil embolization when the patient has coagulopathy (similar to our case), because the thera- peutic effect of NBCA does not depend on the coagulation process (10).

Despite the several advantages mentioned above, we first used a microcoil rather than NBCA. The possible complications of NBCA, such as end-organ ischemia or nontarget embolization, would have increased the patient burden considerably if the pseudoaneurysm was combined with an underlying ECF. Percutaneous thrombin injection was selected as not only an effective treatment with a low complication rate, but also a minimally invasive procedure with no radiation exposure. During the patient's hospital stay, TPN and gradual shift to a fluid diet could successfully control the underlying ECF. We believe that this regimen contributed to the good outcomes of TAE using NBCA, without any associated complications.

Proper treatment should be decided when dealing with pseudoaneurysm, depending on not only the characteristics of the target lesion, such as the lesion size, location, and presence of outflow or collateral vessels (10), but also the patient's comorbidities and medical history, which can affect the rebleeding risk or recurrence. To the best of our knowledge, in the limited number of IEA pseudoaneurysms reported so far, there is no case of IEA 



Figure 2. The follow-up contrast medium-enhanced CT scan (A) indicates a remaining pseudoaneurysm (arrow) within the anterior abdominal wall hematoma, arising from the previous coil embolization site (arrowhead). The Doppler sonogram (B) shows a remaining pseudoaneurysm and perilesional acute hematoma. C, Complete thrombosis of pseudoaneurysm is achieved after percutaneous thrombin injection (900 IU). The follow-up Doppler sonogram (D) indicates a recurrent pseudoaneurysm with a classic "to-and-fro" sign. The superselective left IEA angiogram (E) shows a pseudoaneurysm (arrow) located distal to the previous coil embolization site. F, The NBCA and lipiodol mixture is infused into the pseudoaneurysm. G, The completion angiogram shows the total occlusion of the pseudoaneurysm. 
pseudoaneurysm accompanied by postsurgical ECF, managed by endovascular and percutaneous treatments. Our case showed that an accompanying ECF could increase the unresponsiveness of IEA pseudoaneurysms to treatment by creating an inflammatory environment; therefore, repeated therapeutic trials with different embolic agents or methods might be needed to reach total occlusion.

In conclusion, our findings suggest that a coexisting ECF can affect the unresponsiveness of patients with IEA pseudoaneurysm to widely accepted treatments, such as coil embolization and thrombin injection by creating an inflammatory environment. In such cases, repeated therapeutic trials might be needed. TAE using NBCA can be an effective therapeutic option for patients with refractory IEA pseudoaneurysms combined with ECF.

\section{Footnotes}

Authors' Contributions: Study concept and design, C. Park; Acquisition of data, M.W. Song; Analysis and interpretation of data, N.A.; Drafting of the manuscript, M.W. Song; Critical revision of the manuscript for important intellectual content, B.C. Lee; Statistical analysis, N.A.; Administrative, technical, and material support, N.A.; and Study supervision, H.O. Kim.

Conflict of Interests: There was no conflict of interest.

Ethical Approval: Ethical approval for this study was waived by the institutional review board of our hospital.

Funding/Support: This study received no specific funding support.

Informed Consent: The need to obtain informed consent was waived by the ethics committee.

\section{References}

1. Edwards KJ, Avula SK, Babu BD, England RA. Inferior epigastric artery pseudoaneurysms. Ann R Coll Surg Engl. 2015;97(4):255-8. doi: 10.1308/003588414X14055925058076. [PubMed: 26263930]. [PubMed Central: PMC4473860].

2. Park SW, Choe WH, Lee CH, Lee MW, Kim YJ, Kwon SY, et al. Transcatheter embolization of a pseudoaneurysm of the inferior epigastric artery with N-butyl cyanoacrylate. Br J Radiol. 2008;81(963):e64-7. doi: 10.1259/bjr/86813899. [PubMed: 18270285].

3. Shabani AG, Baxter GM. Inferior epigastric artery pseudoaneurysm: Ultrasound diagnosis and treatment with percutaneous thrombin. Br J Radiol. 2002;75(896):689-91. doi: 10.1259/bjr.75.896.750689. [PubMed: 12153944].

4. Galie KL, Whitlow CB. Postoperative enterocutaneous fistula: When to reoperate and how to succeed. Clin Colon Rectal Surg. 2006;19(4):23746. doi: 10.1055/s-2006-956446. [PubMed: 20011327]. [PubMed Central: PMC2780112].

5. Tonolini M, Magistrelli P. Enterocutaneous fistulas: A primer for radiologists with emphasis on CT and MRI. Insights Imaging. 2017;8(6):53748. doi: 10.1007/s13244-017-0572-3. [PubMed: 28963700]. [PubMed Central: PMC5707219].

6. Sobkin PR, Bloom AI, Wilson MW, LaBerge JM, Hastings GS, Gordon $\mathrm{RL}$, et al. Massive abdominal wall hemorrhage from injury to the inferior epigastric artery: A retrospective review. J Vasc Interv Radiol. 2008;19(3):327-32. doi: 10.1016/j.jvir.2007.11.004. [PubMed: 18295690].

7. Sheiman RG, Mastromatteo M. Iatrogenic femoral pseudoaneurysms that are unresponsive to percutaneous thrombin injection: Potential causes. AJR Am J Roentgenol. 2003;181(5):1301-4. doi: 10.2214/ajr.181.5.1811301. [PubMed: 14573423].

8. Krokidis M, Hatzidakis A, Petrakis J, Lagoudis T, Tsetis D. Coil embolization of inferior epigastric artery pseudoaneurysm after percutaneous thrombin injection failure: A case report. Cases J. 2009;2:6562. doi: 10.4076/1757-1626-2-6562. [PubMed: 19918533]. [PubMed Central: PMC2769303].

9. Wu L, Ren J, Liu Q, Wang G, Wu X, Gu G, et al. Risk factor and outcome for intra-abdominal bleeding in patients with enterocutaneous fistula. Medicine (Baltimore). 2016;95(47). e5369. doi: 10.1097/MD.0000000000005369. [PubMed: 27893673]. [PubMed Central: PMC5134866].

10. Madhusudhan KS, Venkatesh HA, Gamanagatti S, Garg P, Srivastava DN. Interventional radiology in the management of visceral artery pseudoaneurysms: A review of techniques and embolic materials. Korean J Radiol. 2016;17(3):351-63. doi: 10.3348/kjr.2016.17.3.351. [PubMed: 27134524]. [PubMed Central: PMC4842855]. 